RECYT

Año 21 / № 32 / 2019 / 82-86

\title{
Evaluación de la calidad del agua del embalse Mbói Caé
}

\section{Water quality assessment of the Mboi Cae reservoir}

\author{
J. Weseluk ${ }^{1, *}$, F. Vergara1 ${ }^{1}$, V. Berestovoy ${ }^{2}$ \\ 1- Escuela de Posgrado, Universidad Nacional de Itapúa, Encarnación, Paraguay \\ 2- Facultad de Ciencias y Tecnología, Universidad Nacional de Itapúa, Encarnación, Paraguay \\ * E-mail: jcweseluk@gmail.com
}

\section{Resumen}

La modificación del régimen hidrológico del arroyo Mbói Caé constituyó un cambio importante en la ciudad de Encarnación y alrededores que ha beneficiado a las poblaciones con la generación de energía hidroeléctrica, las actividades recreativas, la disponibilidad de agua, etc. La presente investigación tiene el objetivo de analizar la calidad del agua del embalse Mbói Caé del departamento de Itapúa mediante el Índice de Calidad de Agua según el Sistema Holandés de Valoración y el índice de Contaminación ICOTRO basado en la concentración de fósforo total en el periodo noviembre del año 2017 a septiembre del año 2018. Los resultados concluyeron que, teniendo en cuenta los parámetros de oxígeno disuelto, demanda bioquímica de oxígeno y nitrógeno amoniacal, el área de estudio se encuentra categorizada con contaminación incipiente y, por otra parte, se caracteriza como eutrófico por fósforo.

Palabras clave: Calidad de agua; Demanda bioquímica de oxígeno; Fósforo total; Nitrógeno amoniacal; Oxígeno disuelto.

\section{Abstract}

The modification of the hydrological regime of the Mbói Caé stream was an important change in the city of Encarnación and surroundings benefiting the populations with the generation of hydroelectric energy, recreational activities, the availability of water, etc. The objective of this research is to analyze the water quality of the Mbói Caé reservoir in the Itapúa department through the Water Quality Index according to the Dutch Valuation System and the ICOTRO Contamination Index based on the total phosphorus concentration in the period November 2017 to September 2018. The results concluded that, taking into account the parameters of dissolved oxygen, biochemical demand of oxygen and ammoniacal nitrogen, the studied area is categorized with incipient contamination and, on the other hand, it is characterized as eutrophic by phosphorus.

Keywords: Ammonia nitrogen; Biochemical oxygen demand; Dissolved oxygen; Total phosphorus; Water quality.

\section{Introducción}

Según el último informe mundial de las Naciones Unidas sobre el desarrollo de los recursos hídricos se espera que el uso doméstico del agua, que representa aproximadamente el $10 \%$ de las extracciones de agua a nivel mundial, aumente significativamente durante el período 2010-2050 en casi todas las regiones del mundo y que la demanda mundial para la producción agrícola y la energía (principalmente alimentación y electricidad), ambas con un uso intensivo de agua, aumenten aproximadamente un $60 \%$ y un $80 \%$ respectivamente antes de 2025 [19], lo que sumado a las consecuencias del cambio climático supone riesgos en la disponibilidad de agua para las poblaciones; por ello es importante asegurar la calidad a fin de evitar limitaciones en sus usos [20].

La disponibilidad de agua dulce constituye uno de los problemas más críticos referente a recursos naturales, y la construcción de embalses ha sido un método utilizado frecuentemente para garantizar la disponibilidad del agua; sin embargo se debe tener en cuenta que para lograr su sostenibilidad es necesario mantener su calidad [3].

En los últimos años, la cantidad de embalses construidos por el hombre ha aumentado debido a la necesidad de abastecer de agua, alimentos y energía a la población. Cuando en un sistema fluvial se sitúan varios embalses en la misma cuenca, se origina un efecto en cascada por la liberación del agua río abajo, produciendo modificaciones en la calidad del agua [15].

Los índices de calidad de agua son herramientas que permiten diagnosticar la calidad de agua de un cuerpo superficial o subterráneo en un tiempo determinado; por medio de ellos se realiza un análisis general de la calidad del agua en diferentes niveles, y se determina la vulnera- 
bilidad del cuerpo frente a amenazas potenciales. Actualmente existen diferentes metodologías para calcular estos índices, las que se diferencian en las formas de cálculo y en los parámetros utilizados [5].

Uno de los índices utilizado es el "Índice holandés de valoración de la calidad para los cuerpos de agua superficiales" que se basa en la obtención de un puntaje de acuerdo a varios parámetros analizados: la demanda bioquímica de oxígeno $\left(\mathrm{DBO}_{5}\right)$, el oxígeno disuelto (OD) y el nitrógeno amoniacal $\left(\mathrm{N}-\mathrm{NH}_{4}^{+}\right)$[17].

El parámetro de demanda bioquímica de oxígeno $\left(\mathrm{DBO}_{5}\right)$ es una medida del oxígeno consumido en la oxidación de la materia orgánica por acción bacteriana e indica la cantidad de materia orgánica vertida en un cuerpo de agua [6].

El oxígeno es fundamental para la mayoría de los organismos vivos dada su dependencia del proceso de respiración aeróbica para la generación de energía y para la movilización del carbono en la célula; por ello se considera una de las variables más importante para el estudio de la calidad del agua [2]. En los embalses, la concentración de oxígeno disuelto varía dependiendo del incremento de la productividad biológica como resultado de la entrada de nutrientes y de materia orgánica, del patrón de distribución térmica, de su estado trófico, de su ubicación geográfica, morfometría y condiciones meteorológicas [3].

El N-Amoniacal es otro parámetro para diagnosticar la calidad del agua, puesto que en su forma libre como amoniaco es tóxico para los peces; y esta toxicidad depende del $\mathrm{pH}$ y temperatura del agua. Su presencia es relacionada a la descarga directa o a la degradación de la materia orgánica nitrogenada [6].

Este índice mencionado es utilizado como metodología para determinar la calidad del agua en Costa Rica por Decreto Ejecutivo No 33903 del año 2007, en su artículo 19 establece que "La calidad físico-química y biológica de un cuerpo de agua superficial se establecerá haciendo uso del Índice de Clasificación Holandés de Valoración y el Índice Biológico" [12].

Los Índices de Calidad del Agua han sido importantes en el contexto ecológico y medio ambiental pero presentan desventajas relacionadas con la falta de información, por ello se desarrollaron los Índices de Contaminación, los cuales son complementarios en sentido ecológico y permiten precisar problemas ambientales [13].

Uno de estos índices es el ICOTRO o Índice de Contaminación Trófico que se calcula en base a la concentración de fósforo total en las aguas [13].

El fósforo es un nutriente esencial para los organismos vivientes y es considerado como un parámetro crítico en la calidad de aguas debido a su influencia en el proceso de eutrofización [16].

Los principales factores que regulan el crecimiento de las malezas acuáticas son la luz y los nutrientes: y entre estos los que más regulan el crecimiento de las plantas acuáticas son el carbono, el nitrógeno y el fósforo. El fósforo es considerado el más influyente en el crecimiento de las plantas; una pequeña adición de fósforo al agua resulta en un aumento considerable en la biomasa de las plantas [1].

El fósforo se relaciona con el proceso de eutrofización y generalmente se debe a la presencia del contaminante en la descarga cloacal [11].

El nitrógeno y el fósforo en el agua del embalse de Danjiangkou, China provienen de sus afluentes, y por lo tanto, las medidas más efectivas para prevenir y curar la proliferación de algas y la eutrofización del agua es controlar la cantidad total de contaminantes en cada uno de estos afluentes [9].

En un estudio de caso de las corrientes de agua que alimentan el reservorio de agua potable de Zelivka, República Checa, se encontró que el fósforo era el nutriente limitante para el crecimiento del fitoplancton y, por lo tanto, se lo atribuyó como factor principal responsable de las tasas de eutrofización del reservorio, y que probablemente podría controlarse de manera efectiva mediante acciones concertadas de reducción [8].

Un estudio en el reservorio Saguling de Indonesia muestra que la concentración de fósforo del reservorio fue de $315.0 \mathrm{mg} \cdot \mathrm{m}^{-3}$ en promedio en 1999-2013 [10].

Los resultados de un estudio de cinco reservorios durante la temporada de riego de 1995 en la parte baja de la cuenca del río South Platte de Estados Unidos mostraron que los reservorios atrapaban del 20 al 88\% del nitrógeno y el fósforo entrantes, excepto el fósforo en un reservorio. Las concentraciones totales de nitrógeno en los reservorios fueron más elevadas en marzo y disminuyeron hasta septiembre, en gran parte como resultado de la captación de algas y otra vida acuática presente [18].

El presente trabajo se centra en la determinación del Índice de Calidad basado en la metodología de Clasificación del Sistema Holandés y la determinación del Índice de Contaminación ICOTRO en el sub-embalse Mbói Caé.

\section{Materiales y Métodos}

\section{Ubicación del área de estudio}

El área de estudio corresponde a la cuenca baja de la cuenca Mbói Caé del departamento de Itapúa cuya subcuenca identificada, según la base de datos de hidrología analítica (AHD), con el número 315173200 y una superficie total de $10.855 \mathrm{~km}^{2}$ [4].

De los $291 \mathrm{Km}^{2}$ de la cuenca del Mbói Cae, $52.7 \mathrm{~km}^{2}$ corresponde a montes nativos, campo libre (pastura) corresponde $160.56 \mathrm{~km}^{2}$, cultivos a $41.11 \mathrm{~km}^{2}$, agua a 6.16 $\mathrm{km}^{2}$ y zona urbana $31.13 \mathrm{~km}^{2}$ [7].

En la Figura 1 se observan los tres puntos (ESM1, ESM2 y ESM3) en donde fue analizado el Oxígeno 


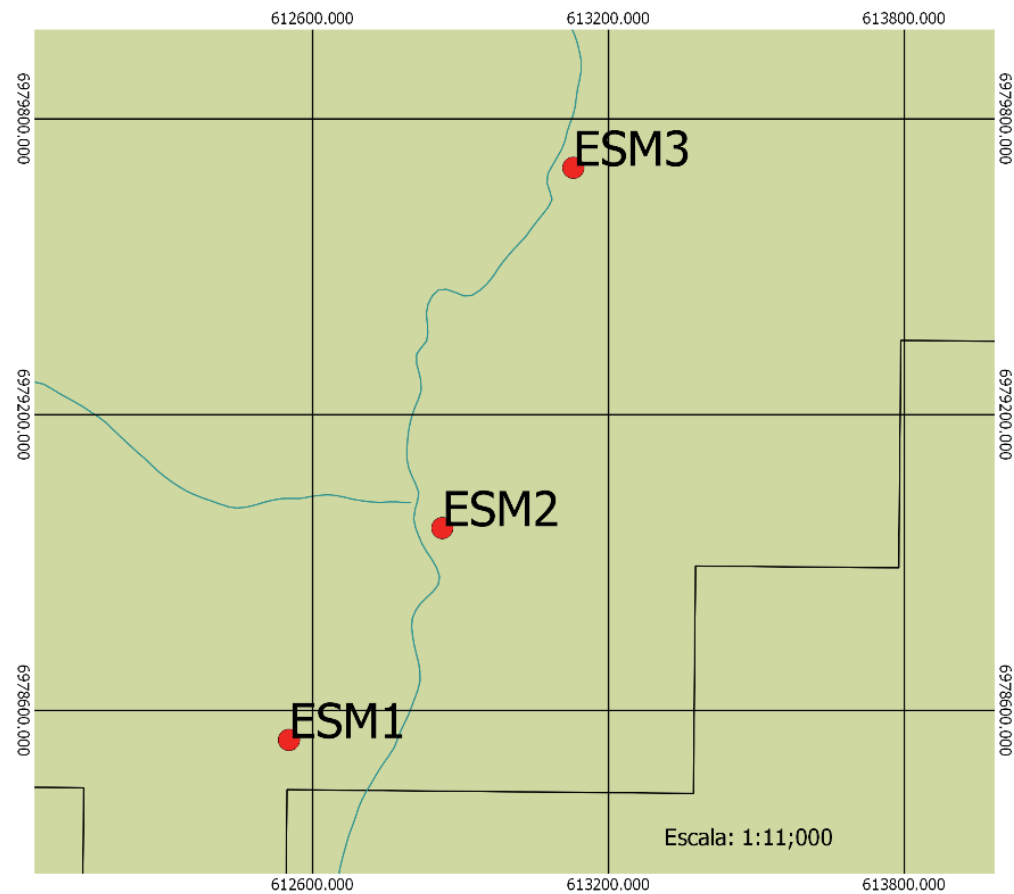

Cuenca Mbói Caé Departamento de Itapúa

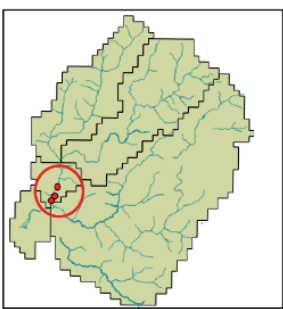

Coordenadas geográficas de los puntos de muestreo

\begin{tabular}{|l|l|l|}
\hline Punto & Latitud & Longitud \\
\hline ESM1 & -27.311589 & -55.862468 \\
\hline ESM2 & -27.307682 & -55.859365 \\
\hline ESM3 & -27.301061 & -55.856745 \\
\hline
\end{tabular}

Figura 1: Ubicación de los puntos de muestreo de fósforo total, oxígeno disuelto, demanda bioquímica de oxígeno y nitrógeno amoniacal en la cuenca Mbói Caé.

Disuelto (sensor de oxígeno), la Demanda Bioquímica de Oxígeno (SM 5210 B), el Nitrógeno Amoniacal (NesslerNN) y el Fósforo Total (SM 4500-P B y SM 4500-P E) mensualmente durante el periodo noviembre 2017 a septiembre 2018.

Estos análisis fueron realizados bajo dirección de la Entidad Binacional Yacyretá dentro del marco que contempla el Plan de monitoreo de los sub-embalses de margen derecha del arroyo Mboi Caé.

\section{ICA Holandés}

Según la metodología del código holandés de clasificación por colores, la calidad en términos de estas variables se pondera con una puntuación asignada para cada uno de los parámetros (Porcentaje de Saturación de Oxígeno, $\mathrm{DBO}_{5}, \mathrm{~N}$-Amoniacal) en función a los datos que se muestran en la Tabla 1. Según el valor de cada parámetro se asigna el puntaje correspondiente, luego se suman los puntos de cada parámetro y el resultado se compara con los datos de la Tabla 2 [6].
Tabla 1: Puntuación asignada para cada uno de los parámetros en función a su concentración.

\begin{tabular}{|c|c|c|c|}
\hline Puntos & PSO, \% & $\mathrm{DBO}_{5}, \mathrm{mg} / \mathrm{l}$ & $\mathrm{N}-\mathrm{NH}^{+4}, \mathrm{mg} / \mathrm{l}$ \\
\hline 1 & $91-100$ & $<=3$ & $<0.50$ \\
\hline \multirow{2}{*}{2} & $71-90$ & \multirow{2}{*}{$3.1-6.0$} & \multirow{2}{*}{$0.50-1.0$} \\
\hline & $111-120$ & & \\
\hline \multirow{2}{*}{3} & $51-70$ & \multirow{2}{*}{$6.1-9.0$} & \multirow{2}{*}{$1.1-2.0$} \\
\hline & $121-130$ & & \\
\hline 4 & $31-50$ & $9.1-15$ & $2.1-5.0$ \\
\hline 5 & $<=30 y>130$ & $>15$ & $>5.0$ \\
\hline
\end{tabular}

Fuente: Calvo \& Mora, 2007.

Tabla 2: Categorización asignada como calidad de agua según la puntuación obtenida.

\begin{tabular}{|c|c|c|c|}
\hline Clase & $\begin{array}{l}\text { Promedio de } \\
\text { puntos }\end{array}$ & $\begin{array}{l}\text { Código de } \\
\text { color }\end{array}$ & $\begin{array}{l}\text { Interpretación } \\
\text { de calidad }\end{array}$ \\
\hline 1 & 3 & Azul & Sin contaminación \\
\hline 2 & $4-6$ & verde & $\begin{array}{l}\text { Contaminación } \\
\text { incipiente }\end{array}$ \\
\hline 3 & $7-9$ & Amarillo & $\begin{array}{c}\text { Contaminación } \\
\text { moderada }\end{array}$ \\
\hline 4 & $10-12$ & Anaranjado & Contaminación severa \\
\hline 5 & $13-15$ & Rojo & $\begin{array}{l}\text { Contaminación } \\
\text { muy severa }\end{array}$ \\
\hline
\end{tabular}

Fuente: Calvo \& Mora, 2007. 


\section{ICOTRO: Índice de contaminación trófico}

El ICOTRO se fundamenta en la concentración de fósforo total que define por sí misma una categoría discreta detallada en la Tabla 3 [13].

Tabla 3: Categorización del Índice de Contaminación según la concentración de fósforo total

\begin{tabular}{|c|c|}
\hline Categoría & Valor \\
\hline Oligotrófico & $<0.01 \mathrm{~g} \cdot \mathrm{m}^{3}$ \\
\hline Mesotrófico & $0.01-0.02 \mathrm{~g} \cdot \mathrm{m}^{3}$ \\
\hline Eutrófico & $0.02-1 \mathrm{~g} \cdot \mathrm{m}^{3}$ \\
\hline Hipereutrófico & $>1 \mathrm{~g} \cdot \mathrm{m}^{3}$ \\
\hline
\end{tabular}

Fuente: Ramírez, et al., 1997

Según la Resolución 222/02 que establece el padrón de calidad de agua del territorio paraguayo, la concentración de fósforo admisible para aguas de clase 2 es de $0,05 \mathrm{mg} / \mathrm{L}$ [14].

\section{Resultados y discusión}

\section{ICA Holandés}

En la Tabla 4 se detallan los códigos de color obtenidos para cada punto de muestreo en cada mes de estudio.

El punto ESM1 y ESM3 presenta en el 73\% de los meses analizados contaminación incipiente y el punto ESM2 presenta la categorización de contaminación incipiente en un $55 \%$.

Las variaciones entre las categorías de "contaminación incipiente" y "sin contaminación" se dan por los cambios en el valor del parámetro de Porcentaje de Saturación de Oxígeno; es decir la variación de este parámetro es lo que diferenció las categorizaciones otorgadas por el método utilizado.

Esta variación puede atribuirse a la vegetación acuática presente en la zona que aumenta en algunos meses dependiendo de la temperatura de ambiente principalmente.

Tabla 4: Categorización de ICA basado en el método holandés obtenido para los tres puntos de estudio en el periodo noviembre 2017 a septiembre 2018.

\begin{tabular}{|c|c|c|c|}
\hline MES & ESM1 & ESM2 & ESM3 \\
\hline \multicolumn{4}{|l|}{ nov-17 } \\
\hline \multicolumn{4}{|l|}{ dic-17 } \\
\hline \multicolumn{4}{|l|}{ ene-18 } \\
\hline \multicolumn{4}{|l|}{ feb-18 } \\
\hline \multicolumn{4}{|l|}{ mar-18 } \\
\hline \multicolumn{4}{|l|}{ abr-18 } \\
\hline \multicolumn{4}{|l|}{ may-18 } \\
\hline \multicolumn{4}{|l|}{ jun-18 } \\
\hline \multicolumn{4}{|l|}{ jul-18 } \\
\hline \multicolumn{4}{|l|}{ ago-18 } \\
\hline \multicolumn{4}{|l|}{ sept-18 } \\
\hline \multicolumn{4}{|c|}{ Referencias } \\
\hline & \multirow{2}{*}{\multicolumn{3}{|c|}{$\begin{array}{l}\text { Contaminación incipiente } \\
\text { Sin contaminación }\end{array}$}} \\
\hline & & & \\
\hline
\end{tabular}

ICOTRO

Las categorías obtenidas en cuanto al Índice ICOTRO en los 3 puntos de estudio en cada mes de estudio se resumen en la Tabla 5.

Tabla 5: Categorización obtenida para el índice ICOTRO basado en la concentración de fósforo total en los tres puntos de muestreo de estudio en el periodo noviembre 2017 a septiembre 2018.

\begin{tabular}{|c|l|l|l|}
\hline MES & ESM1 & ESM2 & ESM3 \\
\hline nov-17 & & & \\
\hline dic-17 & & & \\
\hline ene-18 & & & \\
\hline feb-18 & & & \\
\hline mar-18 & & & \\
\hline abr-18 & & & \\
\hline may-18 & & & \\
\hline jun-18 & & & \\
\hline jul-18 & & & \\
\hline ago-18 & & & \\
\hline sept-18 & & & \\
\hline Referencia & Mesotrófico & & \\
\cline { 1 - 2 } & & & \\
\hline \multicolumn{2}{|l|}{} \\
\cline { 1 - 2 }
\end{tabular}

El 94\% de los periodos y puntos analizados constituyen la categoría "eutrófico" en la zona analizada.

Las principales actividades de la zona son los campos de cultivo y la urbanización. Ambas actividades aportan fósforo por medio de fertilizantes y residuos cloacales.

\section{Conclusiones}

La sub-cuenca aguas abajo de la cuenca Mbói Caé es categorizada con "contaminación incipiente", esta contaminación se da por la variación del parámetro de oxígeno disuelto.

Por otra parte dicha sub-cuenca es categorizada como eutrófica por fósforo, sin embargo los valores se encuentran dentro de lo permisible por la Resolución 222/02 por la cual se establece el padrón de calidad de aguas en el territorio paraguayo.

\section{Bibliografía}

1. Acosta-Arce, L., \& Agüero-Alvarado, R. (2006). Malezas acuáticas como componentes del ecosistema. Agronomía Mesoamericana, 17 (2), 213-218. Disponible en: http:// www.redalyc.org/pdf/437/43717209.pdf

2. Arroyo, V., Millán, C., Castillo, A. \& Aguilar, A. (2012). Acoplamiento de mecanismos de DBO en un modelo CFD para cuerpos de agua. Instituto Mexicano de Tecnología del Agua. México. Disponible en: http://repositorio.imta.mx/bitstream/handle/20.500.12013/1499/ HC-1209.1.pdf?sequence $=1$ \&isAllowed $=y$ 
3. Betancourt, C. \& Labaut, Y. (2013). La calidad fisicoquímica del agua en embalses, principales variables a considerar. Agroecosistemas Vol.1 N¹: 78-103. Disponible en: https://www.researchgate.net/publication/303256205_La_calidad_fisicoquimica_del_agua en_embalses_principales_variables_a_considerar_Physical-chemical_water_quality_of_reservoirs_a_review_of_the_main_variables

4. BID. 2016. Base de datos de hidrología analítica para América Latina y El Caribe. Disponible en: http:// sp.hydrobidlac.org/events/?id=111

5. Caho, c. \& López, E. (2017). Determinación del Índice de Calidad de Agua para el sector occidental del humedal Torca-Guaymaral empleando las metodologías UWQI y CWQI. Producción + Limpia Vol.12, No.2 35-49 - DOI: 10.22507/pml.v12n2a3. Disponible en: http://www.scielo.org.co/pdf/pml/v12n2/1909-0455pml-12-02-00035.pdf

6. Calvo, G. \& Mora, J. (2007). Evaluación y clasificación preliminar de la calidad de agua de la cuenca del río Tárcoles y el Reventazón. Parte III: Calidad de cuerpos receptores de agua, según el Sistema Holandés de Valoración. Tecnología en Marcha Vol. 20-4. Disponible en: http://revistas.tec.ac.cr/index.php/tec_marcha/ article/view/452

7. Global Consultores (2015). Informe final: Balance hídrico Cuenca del arroyo Mbói Caé Encarnación. Entidad Binacional Yacyretá.

8. Holas, J., Holas, M. \& Chour, v. (1999). Pollution by phosphorus and nitrogen in water streams feeding the Zelivka drinking water reservoir. Water Sci Technol (1999) 39 (12): 207-214. Doi: [https://doi.org/10.1016/S02731223(99)00337-6]. Disponible en: https:/www.sciencedirect.com/science/article/pii/S0273122399003376

9. Liu, Y., Zhu, Y., Qiao, X., Zheng, B., Chang, S. \& Fu, Q. (2018). Investigation of nitrogen and phosphorus contents in water in the tributaries of Danjiangkou Reservoir. Royal Society Open Science, 5 (1). doi: [10.1098/rsos.170624] Disponible en: https://www.ncbi.nlm.nih.gov/pmc/articles/PMC5792870/

10. Marselina, M. \& Burhanudin, M. (2018). Phosphorus load concentration in tropical climates reservoir for each water quantity class. Journal of Water and Land Development. 36. 99-104. 10.2478/jwld-2018-0010. Disponible en: https:/www.researchgate.net/publication/324178661_Phosphorus_load_concentration_in tropical_climates_reservoir_for_each_water_quantity_class

11. Plan Encarnación Más. (2014). Plan de desarrollo sustentable y plan de ordenamiento urbano y territorial. Diagnóstico y objetivos. Encarnación, Paraguay.

12. Presidencia de la República de costa Rica. (9 de marzo del 2007). Decreto Ejecutivo $N^{\circ} 33903$ : Reglamento para la Evaluación y Clasificación de la Calidad de los Cuerpos de Agua Superficiales. Disponible en: http:// www.digeca.go.cr/sites/default/files/de-33903reglamento_evaluacion_clasificacion_cuerpos_de_agua_0. pdf

13. Ramírez, A., Restrepo, R. \& Viña, G. (1997). Cuatro índices de contaminación para caracterización de aguas continentales. Formulaciones y aplicaciones. CT\&F - Ciencia, Tecnología y Futuro, 1(3), 135-153. Disponible en: http://www.scielo.org.co/scielo.php?script=sci_arttext \&pid=S0122-53831997000100009

14. Secretaría del Ambiente de Paraguay. (22 de abril del 2002). Resolución $\mathrm{N}^{\circ} 222$ por la cual se establece el padrón de calidad de aguas en el territorio nacional. Disponible en: http://archivo.seam.gov.py/sites/default/files/ resolucion_222_02.pdf

15. Saldaña, M., Díaz, E. \& Gutiérrez, A. (2011). Diagnóstico de la calidad del agua en un sistema de embalses en cascada, cuenca del río San Juan, Querétaro, México. Tecnología y ciencias del agua Vol. $2 \mathrm{~N}^{\circ}$ 3. Versión Online ISSN 2007-2422. Disponible en: www. scielo.org. $\mathrm{mx} /$ scielo.php?script $=$ sci_arttext\&pid $=$ S2007-24222011000300008

16. Sánchez, J. (2001). El fósforo, parámetro crítico de calidad de agua. Técnicas analíticas y de muestreo. Disponible en: http://www.ingenieroambiental.com/junio/fosforo.pdf

17. Serna, J., Sánchez, J. \& Rodríguez, J. (2016). índices de calidad en cuerpos de agua superficiales en la planificación de los recursos hídricos. Revista Logos Ciencia \& Tecnología ISSN 2145-549X | ISSN 2422-4200, Vol. 8, N. 1. Disponible en: https:/www.researchgate.net/publication/311864221_Indices_de_calidad_en_cuerpos_de_ agua_superficiales_en_la_planificacion_de_los_recursos_hidricos

18. Sprague, L.A., Kimbrough, R.A. \& Ranalli, A.J. (2002). What happens to nutrients in offstream reservoirs in the lower South Platte River Basin?. USGS Fact Sheet 044-02. Disponible en: https://pubs.usgs.gov/fs/fs-044-02/pdf/fs044-02.pdf

19. UNESCO. (2018). Informe mundial de las Naciones Unidas sobre el desarrollo de los recursos hídricos 2018: soluciones basadas en la naturaleza para la gestión del agua, cifras y datos. Disponible en: https://unesdoc. unesco.org/ark:/48223/pf0000261579_spa

20. WWAP (Programa Mundial de las Naciones Unidas de Evaluación de los Recursos Hídricos)/ONU-Agua. 2018. Informe Mundial de las Naciones Unidas sobre el Desarrollo de los Recursos Hídricos 2018: Soluciones basadas en la naturaleza para la gestión del agua. París, UNESCO. Disponible en: https://unesdoc.unesco.org/ark:/48223/ pf0000261494

Recibido: 22/03/2019.

Aprobado: 22/05/2019. 